\title{
HYPERSONIC FLOW
}

$\mathrm{O}$ N December 15, some two hundred representatives of the research establishments, the aircraft industry and the universities attended an all-day discussion on hypersonic flow sponsored by the Royal Aeronautical Society and held, under the chairmanship of Dr. G. W. H. Gardner (Royal Aircraft Establishment, Farnborough), in the rooms of the Institution of Mechanical Engineers.

An introductory paper surveying the problems of hypersonic aerodynamics was read by $\mathrm{Mr}$. R. J. Monaghan (Royal Aircraft Establishment, Farnborough). The term 'hypersonic' can generally be applied to flight at Mach numbers greater than about 5 , for it is at speeds of this order that the linearized equations of supersonic flow become inadequate, boundary layers of increased thicknesses begin to interact appreciably with flow fields around slender bodios and aerodynamic heating assumes major importance. A further boundary occurs at Mach numbers of the order of $8-9$, when there is sufficient kinetic energy to provide some dissociation of the oxygen molecules in air, and the amount of dissociation increases with increasing Mach number above this value.

In designing for the alleviation of aerodynamic heating there are two distinct cases. The first relates to the uncontrolled re-entry of a freely falling body, for which a high-pressure drag shape is required; the second refers to sustained flight, for which a shape with low-pressure drag is probably better, radiation giving appreciable control of surface temperature. Low-pressure drag accords with design for aerodynamic efficiency and there is scope for research on three-dimensional lifting shapes.

The strong curved bow wave moving ahead of the body which is a characteristic of hypersonic speed implies that no real distinction can be drawn between blunt and sharp noses; moreover, the necessity for avoiding excessive rates of heat transfer means that the nose must be blunted. Some distinction can be made, however, between bodies in which the curvature varies slowly along the surface, and flatfaced bodies in which a distinct shoulder occurs.

In his paper on general characteristics of hypersonic flow fields, Dr. R. N. Cox (Armaments Research and Development Establishment, Fort Halstead) gave a historical survey of early work on hypersonic flow and discussed the problems of predicting pressure distributions over blunt-nosed slender bodies.

Expressions have been obtained by many authors for predicting these pressure distributions, but unfortunately the estimates do not agree with experimental measurements. It has now become generally accepted that the magnitude of viscous interaction is a function of Reynolds number based on the thickness of the leading edge; above a certain value of this Reynolds number (that is to say, if the leading edge is blunt) the pressures induced near the leading edge of a body are almost entirely due to inviscid flow effects. Afterwards, there had been much theoretical work on the details of inviscid flow fields, and some promising approximate methods have appeared-for example, the use of Newtonian flow followed by a Prandtl-Meyer expansion for flow over round noses, and the application of the intense blast-wave analogy for predicting induced pressures over the rear portion of the body.

Problems of truly blunt bodies were considered by Dr. K. W. Mangler (Royal Aircraft Establishment, Farnborough) in his paper on special aspects of hypersonic flow fields. Following the bow wave ahead of a blunt body is a region of subsonic flow, and very reasonable estimates of the flow variables in this region can be obtained from inviscid theory, provided that allowance is made for the variation of entropy across the shock, and that the equation of state is adjusted to make allowance for the different degrees of freedom excited in the molecules of the gas. Accordingly, consideration was given to a theoretical model of an inviscid flow at very large Mach number and with a ratio of enthalpy to internal energy approaching unity. Attempts to extend this work to cover the more general case of arbitrary large Mach numbers and more realistic values of the enthalpy - internal energy ratio have shown the necessity for adopting numerical techniques.

Many of these techniques deal with the inverse problem of finding the body to fit a given shock shape, which simplifies the procedure considerably, and on these lines a marching technique can be devised. The non-viscous concept gives satisfactory results for the forward-facing surface, but, behind the shoulder of the body, viscosity has to be taken into account, and it is necessary to match the values of the flow properties at the outer edge of the boundary layer with those of the flow entering from the inviscid region.

A paper on hypersonic boundary layers with special reference to aerodynamic heating by $\mathrm{Dr}$. L. F. Crabtree (Royal Aircraft Establishment, Farnborough) emphasized the importance of the leading edge in problems of heat transfer. The rate of heat transfer to a bluff body, including dissociation effects, can be investigated analytically.

The dense layer of air near a highly cooled surface is relatively unaffected by pressure gradients along the surface, a concept which proved very useful in calculations of turbulent boundary layers for blunt bodies; if true local conditions are used, the intermediate enthalpy method is very useful, for undissociated flow, for both laminar and turbulent boundary layers at Mach numbers up to 10 .

Dr. K. W. Mangler had mentioned the problems of lifting bodies with their associated problems of vortex and bubble type separations. From the point of view of low drag the former would appear preferable, but a planned use of the latter also has possibilities; for example, a spike protruding from the nose of a blunt body can reduce the drag. However, difficulties arise in this case, since experiments have shown high heating-rates when the flow becomes re-attached; indeed, the overall heat transfer might be higher than on the basic bluff body without a spike. Other methods have been suggested for the reduction of aerodynamic heating-rates, including ablating surfaces and transpiration cooling; for the latter method, experiment has shown that quite modest amounts of coolant blown through a wall can produce a considerable reduction in the heating-rates. 
Methods of studying experimentally some of the problems of hypersonic flows were surveyed by Dr. D. W. Holder (National Physical Laboratory, Teddington). Some of the new problems can be studied with techniques which do not differ in principle from those used in research at supersonic speeds. However, to simulate fully the very high temperatures of hypersonic flight it is usually necessary to employ heaters or tunnels of unconventional design, frequently having a very short running time, or to make tests with a model launched or propelled at a very high velocity. In such tunnels the working gas is usually heated by compression, in a shock tube or by a system of shock waves, or by the discharge of electrical energy.

In addition to the usual wind-tunnel measurements of aerodynamic loading and heat transfer, the pressure, temperature and density of the working gas are required ; the degrees of dissociation and ionization and their variation with temperature and time are other properties which have to be measured within the brief period of steady flow provided by the test facility.

Types of facility at present in use include helium tunnels, arc-heated and plasma jets, hypersonic guntunnels, shock tubes, shock tunnels, 'hot-shot' aredriven tunnels, free-flight wind-tunnels and free-flight rocket-propelled models; none of these is able to simulate fully all the required conditions, but each is able to assist in investigation of the problems of some part of the hypersonic regime.

Prof. M. J. Lighthill (University of Manchester) began his paper by a detailed study of the dynamics of dissociating oxygen. Two mechanisms of dissociation exist, one by collision with another particle, the other by absorption of a photon of light. The wave-lengths of light required are in the ultra-violet range (especially 1300-1750 A.), and although at high altitudes the wave-lengths are present in sunlight, they are almost entirely absorbed by the dissociation mechanism. There is practically no light of this wave-length present at altitudes less than $60 \mathrm{~km}$., and at lower altitudes all dissociation is due to collisions.

Conversely, recombination can be effected in two ways: three-body recombination and two-body combination with emission of light. The latter predominates at very low densities, say, less than $10^{-8} \mathrm{gm} . / \mathrm{cm}^{3}$. Where aerodynamic forces are important, three-body recombination is more likely to occur.

The energy required to dissociate an oxygen molecule can be described as the kinetic energy that it would have if its speed were $5.5 \mathrm{~km}$. $/ \mathrm{sec}$. Thus, some dissociation will occur around any body travelling through oxygen at this speed. Not all molecules will be dissociated, for some of the energy will go to increase the translational, vibrational and rotational energy of the molecules.

On passing through the shock wave ahead of the body, the kinetic energy of the molecule is converted almost entirely to translational and rotational energy, and a transitional region exists before dissociational equilibrium is reached; however, equilibrium is reached so rapidly that no direct measurements of satisfactory accuracy have been made in this region.

During dissociation the pressure increases, and this has the effect of compressing the air cap between the shock wave and the body. At very low densities the thickness of the boundary layer is comparable with that of the compressed air cap, and it has been concluded that if the surface were cooled to a temperature not much greater than that of the free stream, then the cooled boundary layer would have an effective negative displacement thickness.

A paper by Dr. J. A. Shercliff (University of Cambridge) on possible aeronautical applications of magnetogasdynamics began by stating that, in the equilibrium state, the ionization process is amenable to the same thermodynamic and statistical-mechanical treatment as the dissociation process. However, it is possible in hypersonic flow that ionization, like dissociation, can fail to reach equilibrium, and the ionization relaxation process behind a bow shock wave can occupy a region comparable in extent with the dimensions of the vehicle.

Magnetogasdynamics is generally understood to exclude the type of electron behaviour which belongs to plasma physics, and the thermodynamics of the gas is considered to be unaffected by the magnetic field.

The interaction of velocity fields with magnetic fields produces the novel features of magnetogasdynamies, and many parallels with fluid dynamics exist-for example, the so-called magnetic Reynolds number measuring the relative power of the convective and diffusive processes. If this is large, a magnetic boundary layer occurs (the skin effect, well known to electrical engineers), but in aero. nautics, magnetic disturbances diffuse much more rapidly than viscous disturbances, and the viscous boundary layer can be ignored when studying the field distortion.

In aeronautics the most obvious application of magnetogasdynamics lies in its use to modify forces on, or heat transfer to, a vehicle. Drag can be produced in midstream away from the surface of a body, heat transfer to stagnation points and skin friction can be reduced by suitably orientated magnetic fields, and transition or separation of the boundary layer can be controlled. An interesting application is that in which an electrical field supplies energy through electrodes to air, accelerating it for propulsive purposes, or the converse case in which air in motion is used to generate electrical energy, which is then supplied to the vehicle to drive auxiliary systems.

The weight penalty will probably confine the use of electromagnetic rockets to interplanetary space vehicles assembled in satellite orbits. Land-based experimental facilities are not affected by the weight penalty and the possibility of high-enthalpy test plant seems attractive. Another possible use lies in the reduction of heat transfer to hypersonic nozzles.

The final paper, on aerodynamic problems of manned space vehicles, was given by Mr. T. R. F. Nonweiler (The Queen's University of Belfast). Any analysis of a project designed to carry a man into orbit and return him safely to Earth must consider the need to limit the accelerations and retardations of his passage and the temperature of his environment. There is no need to fear excessive accelerations in a powered ascent; even with existing fuels a two-stage rocket would not necessarily involve accelerations of more than $10 \mathrm{~g}$.

Descent from an orbit could be effected by the use of a glider vehicle employing aerodynamic lift; an alternative is the so-called 'parachute re-entry' using aerodynamic drag, although whether or not a 'parachute' is employed is immaterial, for if the shape and drag of the vehicle remain constant the deceleration always has the same characteristic 
variation in time, reaching a maximum value of about $8 g$. Deceleration and aerodynamic heating, except in the region of the leading edge, are much reduced by employing lift in the descent, although the provision of lifting surfaces entails an increase in weight of the vehicle and the unusual stability and control characteristics pose interesting problems.

The most critical conditions will probably be encountered not in the return from a successfully accomplished orbit, but in emergency re-entry from an abortive ascent. In meeting this contingency there appears to be some advantage in the glider type of vehicle rather than the zero-lift type, but the evidence is by no means conclusive at present.

The question of a successful landing involves many possibilities. An airfield would be a remarkably elusive target in an unpowered glide which, as a result of emergency or mistake, might be initiated over the Pacific Ocean. Probably the glider would have to be regarded as expendable, the occupant being ejected by capsule at a low altitude.

B. E. Beadle

\section{INTERFACIAL PHENOMENA}

$\mathrm{T}$ HE biennial conference of the Physical Society, on solid state physics, was held during December 18-20 at the Cavendish Laboratory, Cambridge, under the general title of "Interfacial Phenomena". The conference was attended by about two hundred delegates and twenty-eight papers were presented, which fell into three major groups deseribing, respectively, various effects associated with films deposited in vacuum, the nature of solid-liquid interfaces and the formation of electrodeposited films, and the structure and properties of internal surfaces. The conference brought together physicists, physical chemists and metallurgists, each with a different approach to the study of interfacial phenomena.

In introducing the papers on films deposited in vacuum, D. W. Pashley (Tube Investments Laboratory, Hinxton Hall) gave first a brief historical survey of the experimental work on the growth of oriented deposits on substrates. He defined the terms 'epitaxy' and 'misfit' and showed that under some conditions epitaxy only occurred with a misfit of less than 15 per cent, but that in many cases misfits of much larger than 15 per cent could be tolerated. The limitations of the various techniques used for the investigation of epitaxy were then outlined, the great power of the electron microscope being emphasized.

The potentialities of the electron microscope in this field of study were fully exploited by G. A. Bassett (Tubo Investments, Hinxton Hall). His experiments on nucleation, orientation and growth of vacuum-deposited metal films on ionic and metallic substrates showed that an oriented layer of gold of about 10 A. mean thickness, deposited on a singlecrystal silver substrate, formed nuclei of 20-200 A. diameter which grew in size rather than number until a continuous film was formed at a mean deposit thickness of about $50 \mathrm{~A}$. When gold was deposited on rock salt a similar effect was observed, but there the gold nuclei formed preferentially at slip and cleavage steps on the rock salt surface.

Reflexion electron diffraction techniques were used by R. C. Newman (Associated Electrical Industries, Aldermaston), A. Glossop and D. W. Pashley (Tube Investments, Hinxton Hall), to study the deposition of copper on silver maintained at different tempera. tures. They found diffraction patterns characteristic of copper with its normal lattice constant when the mean film thickness was $0.8 \mathrm{~A}$. and the substrate temperature $20^{\circ} \mathrm{C}$., but by increasing this temperature to $300^{\circ} \mathrm{C}$. copper patterns were obtained at a mean film thickness of $0.2 \mathrm{~A}$. These results showed that at above a critical mean thickness of much less than one atomic layer, aggregation of the copper occurred on the substrate surface rather than the formation of a uniform film.

Somewhat different results on the influence of imperfections in the substrate upon the mode of epitaxial growth were presented by W. J. Dunning and P. G. Fox (University of Bristol). They examined, with the optical microscope, the growth of ammonium iodide upon mica and observed a uniform epitaxial film spreading outwards until cleavage steps on the mica were reached and then a pyramidal form of growth of the iodide crystals was initiated. The importance of the substrate orientation upon kinetics of film growth was demonstrated clearly by the experiments of J. D. Lawless (University of Virginia), who had oxidized spherical single-crystals of copper in air and in copper sulphate solutions. Differences in the thickness of oxide film were shown by changes in the interference colours from different regions of the spherical surface. Examination by electron diffraction and electron microscopy showed differ. ences in the preferred orientation of the polycrystalline oxide film on various zones of the single crystal.

The epitaxial growth of ice crystals on various substrates and its possible effect upon the formation of rain were discussed by B. J. Mason (Imperial College of Sicience and Technology, London). $\mathrm{He}_{\mathrm{e}}$ described observations on the nucleation of ice on covellite which showed that after nucleation ice crystals may spread laterally on the surface without thickening. The experimental technique involved observing, with the optical microscope, the interference colours produced by the thin crystals on the cleaved covellite surface, and Dr. Mason illustrated his results with colour micrographs of kaleidoscopic variety.

Two further papers on electron diffraction studies of epitaxial growth were presented by M. Blackman (Imperial College of Science and Technology, London), who discussed the epitaxial polymorphism of thallium and cæsium halides, and H. Wilman (Imperial College), who gave some new examples of epitaxy. He showed many diffraction photographs illustrating the different forms of epitaxial growth on ionic, organic and metallic substrates of deposits condensed in vacuo or formed by chemical action.

A group of papers on the properties of surface films were presented next. J. A. Bennet (British Scientific Instrument Research Association) described an apparatus which enabled him to measure the electrical resistivity of a gold film as it was being deposited on either alumina or on a substrate of bismuth oxide on alumina. He found the resistivity decreased as the thickness of the film increased, an effect which he attributed to changes in continuity 